\title{
Effects of Five Kinds of Drinking Water on the Lifespan of Daphnia Pulex
}

\author{
Guohong* \\ Fujian Medical University, Drinking Water and Health, China \\ *Corresponding author: Guohong, Fujian Medical University, Drinking Water and Health, China
}

\section{ARTICLE INFO}

Received: 慧 May 01, 2020

Published: 幽 May 12, 2020

Citation: Guohong. Effects of Five Kinds of Drinking Water on the Lifespan of Daphnia Pulex. Biomed J Sci \& Tech Res 27(4)-2020. BJSTR. MS.ID.004526.

Keywords: Drinking Water; Daphnia Pulex; The Lifespan

\begin{abstract}
Drinking water is essential for People's daily life. The quality of water largely determines the quality of life, including the lifespan. To explore the effect of five kinds of water, pure water, tap water, mineral water, hydrogen-rich water and filtered water, on lifespan, In this study, the water quality parameters of five kinds of drinking water were determined, including Ph, metasilicic acid content, electrical conductivity, total dissolved solids, and the contents of 13 major metal elements such as calcium, magnesium, iron, zinc, selenium and strontium. Daphnia magna was used as a model organism. Five kinds of water were used as the only domestic water source,and Survival daphnia number was recorded every $24 \mathrm{~h}$ until they all death.Then, the data were analyzed by kaplan-meier analysis. The results showed that the lifespan of daphnia pulex in mineral water group was the longest, hydrogen rich water and filtration water group was the second, pure water and tap water group was the shortest. Strontium and iron in water may play an important role in prolonging the lifespan and the effect varies with different doses. Whether zinc prolongs the life of daphnia fleas is unclear and calcium may have no effect on life extension.
\end{abstract}

\section{Introduction}

Aging and death are the inevitable outcomes of every species and life span is a highly variable historical trait whose variation can be attributed to genetic and environmental factors. Some studies suggest that about a quarter of the difference in life expectancy in developed countries can be attributed to genetic factors [1], but a greater part of the probability is due to environmental factors. The change of life span is accumulative, which is the result of the interaction of one or several complex factors in the body, and often involves a series of physiological and biochemical changes at the molecular, cellular and system levels [2-5]. The rate of aging and the length of life span vary greatly among species and among individuals of the same species. As we know dringking water plays an essential role in the normal functioning of life. But dringking water is not simple, human health is closely affected by its quality. In the book of "water is the best medicine", Dr. F. bateman notes that water can cure thousands of people [6]. It can be seen that highquality water can even be used as medicine to regulate the function and restore the body to health. Many epidemiological studies on long-lived areas have shown that water plays a crucial role in prolonging life, and the differences in $\mathrm{pH}$ of water and mineral content in water will affect the life span [7-11]. Daphnia pulex is one of the species of daphnia. Daphnia has been used as a biological research model in ecology and evolutionary biology for more than a century, well suited to assessing the impact of the environment on a variety of traits $[12,13]$. Water is the direct living environment to Daphnia pulex, which is sensitive to different qualities of water, so it is suitable for studying the influence of different types of drinking water on lifespan.

At present, there are few animal studies on evaluating the effect of drinking water on life span at home and abroad and no studies have been reported to evaluate the effect of potable water on the lifespan of daphnia pulex. However, there are many studies on the influence of diet restriction, resveratrol, maternal age and other external factors on the lifetime of daphnia pulex [14,15]. In this study, pure water, tap water, mineral water, hydrogen-rich water and filtered water were used as research objects, and daphnia daphnia a biological model, we aim to investigate the effects of five. kinds of drinking water on the lifespan of daphnia pulex. 


\section{Materials and Methods}

\section{Materials}

Experimental Animals: Daphnia pulex (college of oceanology, Qingdao agricultural university), after one week of adaptive culture in the laboratory, infant Daphnia pulex (born at the same time) was tested. The daphnia pulex test required starvation culture for 12-24 hours to empty the intestine.

Experimental Apparatus: Beaker, disposable dishes, volumetric flask, BS224S electronic balance (Beijing dolly scientific instrument co., LTD., China), ERC2012 multifunctional water quality analyzer (Beijing east Fang Rui ze trade co., LTD., China), UV9200 ultraviolet spectrophotometer, NexION350 inductively coupled plasma mass spectrometry (icp-ms) (American parkin Elmer company).

Experimental Reagents: Yeast liquor $(30 \mathrm{mg} / \mathrm{ml})$, Ammonium molybdate, $\mathrm{P}$ - nitrophenol indicator, $\mathrm{C}_{2} \mathrm{H}_{2} \mathrm{O}_{4}, \mathrm{NaOH}, \mathrm{HCl}$.

\section{Water for Experiment}

Pure Water: Prepared by cpb-plus direct supply laboratory pure water system (fuzhou dongze medical equipment co., LTD.) of fujian medical university, with conductivity $\leq 5 \mathrm{~s} / \mathrm{m}$.

Mineral Water: Evian mineral water for sale.

Filter Water: Tap water is filtered through a household water purifier, which contains 5 filter elements, namely polypropylene melting and spraying filter element, ceramic composite filter element, compressed activated carbon filter element, composite filter element and compressed activated carbon filter element. Set the group before the boiled water cooling.

Tap Water: The piped water provided by fuzhou municipal water supply system is sampled in the 506 laboratory of school of public health, fujian medical university. Before sampling, open the faucet and let the water flow for about $10 \mathrm{~min}$.

Hydrogen-Rich Water: Cooling the boiled pure water then rich hydrogen cup (PX - $\mathrm{HO}_{2}$ type).

\section{Methods}

\section{Experimental Methods}

Grouping and Treatment of Lifespan Observation: Daphnia pulexs born at the same time were randomly divided into pure water group, mineral water group, tap water group, hydrogen-rich water group and filtered water group. Forty daphnia pulex were set up in each group. The culture temperature of each group was controlled to be the same, between $16^{\circ} \mathrm{C}$ and $25^{\circ} \mathrm{C}$. $3 \mathrm{ml}$ yeast liquor $(30 \mathrm{ml} / \mathrm{L})$ was fed every 24 hours and count the number of survival daphnia pulex, the death and newly bred fleas were sucked out with disposable straw.

Determination of Water Quality Parameters of the Tested Water: Each drinking water collecting water samples from 10 pieces, each piece of measuring cylinder accurately measuring $100 \mathrm{~mL}$, with a multifunctional water quality analyzer determination, detection of indicators include: $\mathrm{pH}$, total dissolved solids(TDS), oxidation - reduction potential(ORP). The determination of $\mathrm{H}_{2} \mathrm{SiO}_{3}$ was carried out by silicon-molybdenum yellow spectrophotometry. The main metal elements were determined in 10 samples of each drinking water by ICP-MS.

Statistical Analysis Method: The experimental data were expressed as $\mathrm{x} \pm \mathrm{s}$, and SPSS 20.0 statistical software was used for data processing and analysis. Kaplan-meier method was used to describe the distribution of survival time. If there was no crossover of survival curve, Log rank test was directly used for comparison between groups. If the survival curves were intersected, survival at each time point was compared using the test or Fisher's exact probability method. $\mathrm{P}<0.05$ was considered statistically significant.

\section{Results}

The Parameter of Five Kind of Dranking Water: As shown in Table 1, hydrogen-rich water, filtered water and mineral water are alkaline water, pure water is neutral water, tap water is weak acid; The content of $\mathrm{H}_{2} \mathrm{SiO}_{3}$ is filtered water > mineral water > tap water, and there is little $\mathrm{H}_{2} \mathrm{SiO}_{3}$ in pure water and hydrogen-rich water; The ORP of hydrogen-rich water and filtered water was negative, indicating that the tow kinds of water are reductive, and the five kinds of water showed hydrogen-rich water $>$ filtered water $>$ mineral water $>$ pure water $>$ tap water. Pure water and hydrogenrich water contain only a very small amount of TDS. The content of TDS in tap water is close to which in filtered water ,more than $80 \mathrm{mg} / \mathrm{L}$ but not more than $100 \mathrm{mg} / \mathrm{L}$. The content of TDS in mineral water is the highest, reaching more than $500 \mathrm{mg} / \mathrm{L}$. Table 2 shows that there are little contents of $\mathrm{Se}, \mathrm{Be}, \mathrm{Co}, \mathrm{Tl}$ and $\mathrm{Cr}$ in five kinds of water, which can't be detected by ICP-MS test. The distribution of other elements in five kinds of water is as follows:Ca: pure water $\approx$ hydrogen-rich water $<$ detection limit $<$ tap water $\approx$ mineral water $<$ filtered water; Zn: pure water $\approx$ hydrogen-rich water $<$ detection limit $<$ mineral water $<$ filtered water $<$ tap water; Sr and Li are: pure water $\approx$ hydrogen-rich water $<$ detection limit $<$ tap water $\approx$ filtered water $<$ mineral water; Fe: pure water $\approx$ hydrogen-rich water $<$ tap water $\approx$ filtered water $<$ mineral water; Mg: pure water $\approx$ rich hydrogen water $<$ detection limit $<$ mineral water $<$ tap water $<$ filtered water; $\mathrm{Cu}$ and $\mathrm{Mn}$ are: mineral water $\approx$ pure water $\approx$ rich hydrogen water $<$ detection limit $<$ tap water $<$ filtered water. 
Table 1: Comparison of general indicators of drinking water quality $(n=10, x \pm s)$.

\begin{tabular}{|c|c|c|c|c|}
\hline Group & $\mathbf{p H}$ & $\left.\mathbf{H}_{2} \mathbf{S i O}_{\mathbf{3}} \mathbf{( m g} / \mathbf{L}\right)$ & $\mathbf{O R P ( m v )}$ & TDS (mg/L) \\
\hline PW & $6.91 \pm 0.24$ & $<1.00$ & $317.12 \pm 7.20$ & $1.00 \pm 0.45$ \\
\hline HW & $7.80 \pm 0.02$ & $<1.00$ & $-304.02 \pm 5.11$ & $1.01 \pm 0.21$ \\
\hline TW & $6.62 \pm 0.13$ & $14.37 \pm 0.23$ & $504.25 \pm 9.00$ & $83.02 \pm 2.01$ \\
\hline FW & $8.10 \pm 0.01$ & $24.32 \pm 0.56$ & $-208.43 \pm 17.14$ & $92.11 \pm 2.94$ \\
\hline MW & $7.30 \pm 0.01$ & $19.11 \pm 0.89$ & $103.03 \pm 6.29$ & $525.89 \pm 6.54$ \\
\hline
\end{tabular}

Note: In table 1, pure water(PW), tap water(TW), mineral water(MW), hydrogen-rich water(HW) and filtered water(FW).

Table 2: Comparison of main metal elements in five kinds of drinking water $(n=10, \mu g / L, x \pm s)$.

\begin{tabular}{|c|c|c|c|c|c|}
\hline Elements & PW & HW & TW & FW & MW \\
\hline $\mathrm{Se}$ & $\leq 0.1$ & $\leq 0.1$ & $\leq 0.1$ & $\leq 0.1$ & $\leq 0.1$ \\
\hline $\mathrm{Ca}$ & $\leq 3.0$ & $\leq 3.0$ & $6.03 \pm 0.56$ & $30.69 \pm 1.25$ & $3.23 \pm 0.32$ \\
\hline $\mathrm{Zn}$ & $\leq 0.1$ & $\leq 0.1$ & $98.43 \pm 20.24$ & $45.65 \pm 1.02$ & $2.22 \pm 2.36$ \\
\hline $\mathrm{Sr}$ & $\leq 0.03$ & $\leq 0.03$ & $11.28 \pm 0.41$ & $9.13 \pm 0.34$ & $451.32 \pm 4.34$ \\
\hline $\mathrm{Fe}$ & $4.17 \pm 0.04$ & $4.27 \pm 0.27$ & $19.27 \pm 1.30$ & $17.36 \pm 1.02$ & $95.30 \pm 2.48$ \\
\hline $\mathrm{Mg}$ & $\leq 0.7$ & $\leq 0.7$ & $0.10 \pm 0.01$ & $0.68 \pm 0.03$ & $0.03 \pm 0.01$ \\
\hline $\mathrm{Cu}$ & $\leq 0.05$ & $\leq 0.05$ & $0.79 \pm 0.23$ & $3.67 \pm 0.27$ & $\leq 0.05$ \\
\hline $\mathrm{Mn}$ & $\leq 0.009$ & $\leq 0.009$ & $1.60 \pm 0.34$ & $2.95 \pm 0.11$ & $\leq 0.009$ \\
\hline $\mathrm{Be}$ & $\leq 0.002$ & $\leq 0.002$ & $\leq 0.002$ & $\leq 0.002$ & $\leq 0.002$ \\
\hline $\mathrm{Co}$ & $\leq 0.001$ & $\leq 0.001$ & $\leq 0.001$ & $\leq 0.001$ & $\leq 0.001$ \\
\hline $\mathrm{Li}$ & $\leq 0.01$ & $\leq 0.01$ & $0.41 \pm 0.02$ & $0.42 \pm 0.01$ & $2.52 \pm 0.03$ \\
\hline $\mathrm{Tl}$ & $\leq 0.1$ & $\leq 0.1$ & $\leq 0.1$ & $\leq 0.1$ & $\leq 0.1$ \\
\hline $\mathrm{Cr}$ & $\leq 0.02$ & $\leq 0.02$ & $\leq 0.02$ & $\leq 0.02$ & $\leq 0.02$ \\
\hline
\end{tabular}

Note: In table 2, pure water(PW), tap water(TW), mineral water(MW), hydrogen-rich water(HW) and filtered water(FW). Undetected results are recorded as less than or equal to the detection limit.

Differences in Lifespan of Daphnia Pulex: Synthesize Figure $\quad:$ pure water group $\approx$ tap water group $<$ hydrogen-rich water 1, which is corresponding to daphnia pulex's survival curve, Table 3, group $\approx$ filtered water group < mineral water group (analysis has timetable and Table 4, Statistical results of pairwise comparison of no statistically significant differences between groups expressed survival between groups, we can find that the lifetimes of daphnia pulex in different kind ofwater aren't exactly the same. It showed in " $\approx$ "). The average survival time and median survival time of the mineral water group were the longest, up to more than 35 days.

Table 3: Survival schedules of daphnia magna.

\begin{tabular}{|c|c|c|c|c|c|}
\hline Group & Number of events & Censoring data & Censoring ratio & $\begin{array}{c}\text { Mean survival time } \\
(\mathbf{x} \pm \mathbf{s}, \mathbf{d a y})\end{array}$ & $\begin{array}{c}\text { Median Lifetime } \\
\text { (day) }\end{array}$ \\
\hline PW & 40 & 0 & 0 & $16.40 \pm 1.36$ & 15.00 \\
\hline TW & 40 & 0 & 0 & $15.50 \pm 1.26$ & 16.00 \\
\hline HW & 40 & 0 & 0 & $29.30 \pm 2.16$ & 30.00 \\
\hline MW & 40 & 0 & 0 & $35.45 \pm 2.50$ & 38.00 \\
\hline FW & 40 & 0 & 0 & $26.07 \pm 2.17$ & 27.00 \\
\hline
\end{tabular}

Table 4: Statistical results of pairwise comparison of survival between groups.

\begin{tabular}{|c|c|c|}
\hline Group & P & Whether the Difference was Statistically \\
Significant
\end{tabular}




\begin{tabular}{|c|c|c|}
\hline 1 与 4 & $<0.001$ & $\mathrm{Y}$ \\
\hline 2 与 3 & 0.037 & $\mathrm{Y}$ \\
\hline 2 与 4 & 0.266 & $\mathrm{~N}$ \\
\hline 3 与 4 & 0.004 & $\mathrm{Y}$ \\
\hline
\end{tabular}

Note: In table 4, “0, 1, 2, 3 and 4" respectively means "pure water group, tap water group, hydrogen-rich water group, mineral water group and filtered water group". "N" means " $\mathrm{NO}$ ", “ $\mathrm{Y}$ ” means “ $\mathrm{NO}$ ".

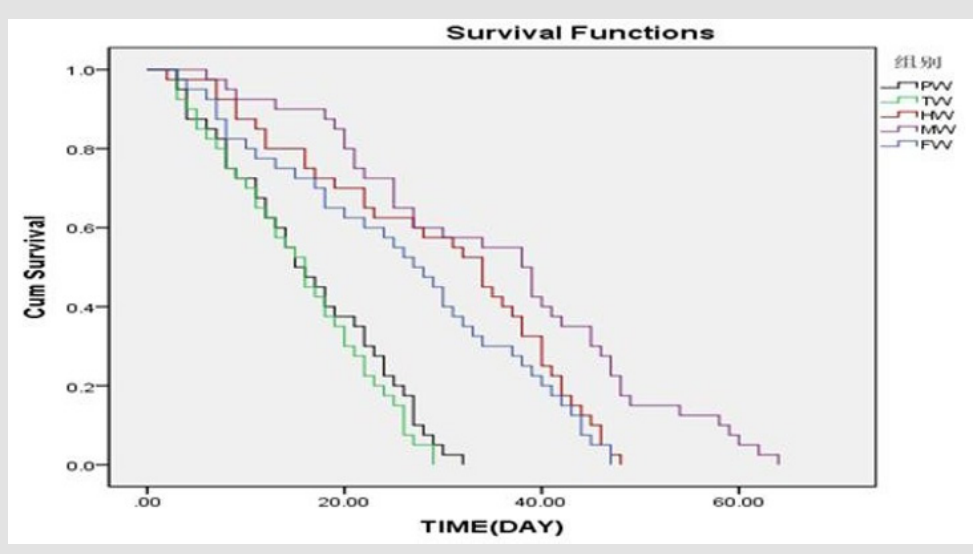

Figure 1: Survival curve of daphnia pulex.

\section{Discussion}

Today, there are various types of water in our daily life. As the development of the study on dranking water in longevity area,we found that there are various factors that decide water quality, such as $\mathrm{pH}, \mathrm{ORP}, \mathrm{TDS}$, content of $\mathrm{H}_{2} \mathrm{SiO}_{3}$ and content of mineral element and so on, had become important factors affecting the quality of drinking water. Immortality has always been the goal of mankind. In ancient times, The emperor tried different pills aimed to be immortal.Today, people are still searching for the secret of longevity in order to prolong human life. In structure, caenorhabditis elegans is Simpler than daphnia pulex, but at present, it has been widely studied as a biological model in life span research, and the research results have been applied to mammals, which has great significance in extending human life span and improving human life quality [16-20]. Compared with caenorhabditis elegans, daphnia pulex has a more complete morphological structure and lives in water. Therefore, It' s reasonable in this study that used daphnia fleas as a model to investigate the effects of five different types of drinking water on the lifespan of daphnia pulex. The parameter analysis results of five different types of water, including: pure water, tap water, filtered water, hydrogen-rich water and mineral water, showed that mineral water, filtered water and hydrogen-rich water are weakly alkaline and have low ORP, among which, the ORP of filtered water and hydrogen-rich water are negative mean they are with reducibility. The results of five groups of daphnia pulex showed that the effects of pure water and tap water on the lifetime of daphnia daphnia were similar. Compared with the pure water group and the tap water group, mineral water, filtered water and hydrogen-rich water extended the lifespan of daphnia daphnia in different degrees. It is preliminarily believed that alkaline water and low ORP water may play a important role in prolongation of life due to its antioxidant activity. Researchers from the world health organization (WHO) and other agencies have discovered that the secret to a long and healthy life lies in drinking raw water rich in mineral nutrients.

In this study, it was found that high iron and high strontium mineral water significantly extended the survival period of daphnia pulex, which reached the longest in the five experimental groups, and the difference was statistically significant. However, there had no advantage in $\mathrm{pH}, \mathrm{H}_{2} \mathrm{SiO}_{3}$ content, ORP for mineral water, it can be seen that strontium and iron seem really play great role in the extended life. From the report about investigation of longevity area we can usually read the discovery of strontium in common water. But whether iron in water plays a role in extending life is unknown, which need further research. The effects of tap water and pure water on the survival of daphnia magna were similar and the difference was not statistically significant. However, the results of metal element analysis showed that the zinc in tap water was significantly higher than that in the pure water group, possibly because the main nutritional role of zinc was to promote growth and development and enhance immunity [21].

It has little effect on life extension or the dose of $98.43 \mu \mathrm{g} / \mathrm{L}$ is too small. It may also due to the high ORP of tap water, which has strong oxidation, can accelerate the aging, which is offset by the effect of zinc. Similarly, there was no statistically significant difference in the lifespan of daphnia pulex between the filtered water group and the hydrogen-rich water group. Both the hydrogen-rich water and the filtered water showed weak alkalinity and strong reduction. The difference was that compared with hydrogen-rich water, the filtered water obviously contained calcium, zinc, strontium and 
iron. According to the analysis of mineral water group, we found strontium and iron in water are likely to play a great role in the life extension. However, the lifespan of daphnia pulex in filtered water did not longer than which in hydrogen-rich water obviously. It may due to the low dose ,so,we speculate that the role of strontium and iron in prolonging life is dose dependent,which need a further study. As for calcium, it may do little to prolong life. In summary, the order of the effects of five drinking water types on the lifespan of D.pulex was as follow, pure water group $\approx$ tap water group < hydrogen rich water group $\approx$ filtered water group $<$ mineral water group (" $\approx$ "means the difference in lifespan has no statistical significance). Strontium and iron in water may play an important role in prolonging the lifespan, which is affected by dose. Whether zinc prolongs the life of daphnia pulex is unclear. Calcium may do little to prolong life.

\section{References}

1. Gusarov I, Pani B, Gautier L, Olga Smolentseva, Svetlana Eremina, et al (2017) Glycogen controls Caenorhabditis elegans lifespan and resistance to oxidative stress. Nature Communications 8: 15868.

2. Andreas H Ludewig, Clotilde Gimond, Joshua C Judkins, Staci Thornton, Dania C Pulido, et al. (2017) Larval crowding accelerates C. elegans development and reduces lifespan. Plos Genetics 13(4): e1006717.

3. Tiku V, Jain C, Raz Y, Shuhei Nakamura, Bree Heestand, et al. (2017) Small nucleoli are a cellular hallmark of longevity. Nature Communications 8: 16038.

4. Xu M, Pirtskhalava T, Farr JN, Bettina M Weigand, Allyson K Palmer, et al (2018) Senolytics improve physical function and increase lifespan in old age. Nature medicine 24(8): 1246-1258.

5. Eline PS, Van NDB, Deelen J (2017) Phenome and genome based studies into human ageing and longevity: An overview. Biochim Biophys Acta 1864(9): 2742-2751.

6. F bateman (2014) Water is the best medicine. Friends of the farmhouse 3: 46

7. Querido W, Rossi AL, Farina M (2016) The effects of strontium on bone mineral: A review on current knowledge and microanalytical approaches. Micron 80: 122-134.

8. Du Y, Luo K, Hussain R (2017) Comparative study of physico-chemical parameters of drinking water from some longevity and non-longevity areas of China. Journal of Water and Health 15(3): 462-473.

9. Fajardo VA, Leblanc PJ, Fajardo VA (2018) Trace lithium in Texas tap water is negatively associated with all-cause mortality and premature death. Applied Physiology, Nutrition, and Metabolism 43(4): 412-414.
10.Wang S, Luo K, Ni R, Yuan Tian (2017) Assessment of elemental background values and their relation with lifespan indicators: A comparative study of Jining in Shandong Province and Guanzhong area in Shaanxi Province, northern China. The Science of the total environment 265(3): 315-324

11. Cai D, Li D, Zhao S, Xiaowei Dou, Fang Wang, et al. (2015) A correlation between diet and longevity characterization by means of element profiles in healthy people over 80 years from a Chinese longevous region. Biological trace element research 165(1): 18-29.

12. Kim E, Ansell CM, Dudycha JL (2013) Resveratrol and food effects on lifespan and reproduction in the model crustacean Daphnia.. Journal of Experimental Zoology Part A Ecological \& Integrative Physiology 321(1): 48-56.

13. Dudycha JL, Brandon CS, Deitz KC (2012) Population genomics of resource exploitation: insights from gene expression profiles of two Daphnia ecotypes fed alternate resources. Ecology \& Evolution 2(2): 329-340.

14. Kim E, Ansell CM, Dudycha JL (2013) Resveratrol and food effects on lifespan and reproduction in the model crustacean Daphnia.. Journal of Experimental Zoology Part A Ecological \& Integrative Physiology 321(1): 48-56.

15. Plaistow SJ, Shirley C, Collin H, Stephen J Cornell, Ewan D Harney (2015) Offspring Provisioning Explains Clone-Specific Maternal Age Effects on Life History and Life Span in the Water Flea, Daphnia pulex. The American Naturalist 186(3): 376-389.

16. Fei T, Fei J, Huang F, Tianpei Xie, Jifeng Xu, et al. (2017) The anti-aging and anti-oxidation effects of tea water extract in Caenorhabditis elegans. Experimental gerontology 97(15): 89-96.

17. Laurent Mouchiroud, Laurent Molin, Prasad Kasturi, Mohamed N Triba, Marc Emmanuel Dumas, et al. (2011) Pyruvate imbalance mediates metabolic reprogramming and mimics lifespan extension by dietary restriction in Caenorhabditis elegans. Aging Cell 10(1): 39-54.

18. Kim Zarse, Saskia Jabin, Michael Ristow (2012) l-Theanine extends lifespan of adultCaenorhabditis elegans. European Journal of Nutrition 51(6): 765-768

19. Jakobsen H, Bojer MS, Marinus MG, Tao Xu, Carsten Struve, et al. (2013) The Alkaloid Compound Harmane Increases the Lifespan of Caenorhabditis elegans during Bacterial Infection, by Modulating the Nematode's Innate Immune Response. PLoS ONE 8(3): e60519.

20. Sunitha Rangaraju, Gregory M Solis, Sofia I Andersson, Rafael L Gomez Amaro, Rozina Kardakaris, et al. (2015) Atypical antidepressants extend lifespan of Caenorhabditis elegans by activation of a non-cellautonomous stress response. Aging Cell 14(6): 971-981.

21. Kambe T, Fukue K, Ishida R, Shiho Miyazaki (2015) Overview of Inherited Zinc Deficiency in Infants and Children. Journal of Nutritional Science and Vitaminology 61: S44-S46.
ISSN: 2574-1241

DOI: $10.26717 /$ BJSTR.2020.27.004526

Guohong. Biomed J Sci \& Tech Res

This work is licensed under Creative

Commons Attribution 4.0 License

Submission Link: https://biomedres.us/submit-manuscript.php

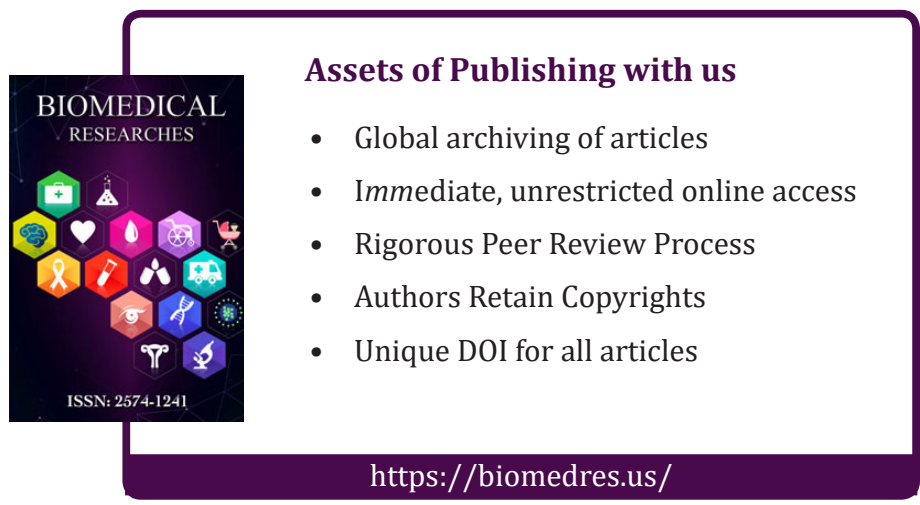

